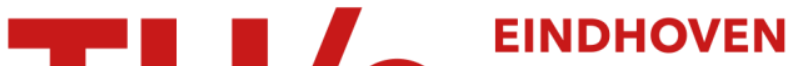 UNIVERSITY OF TECHNOLOGY
}

\section{Self-consistent cluster approach to the homogeneous kinetic nucleation theory}

\section{Citation for published version (APA):}

Kalikmanov, V. I., \& van Dongen, M. E. H. (1993). Self-consistent cluster approach to the homogeneous kinetic nucleation theory. Physical Review E: Statistical, Physics, Plasmas, Fluids, and Related Interdisciplinary Topics, 47(5), 3532-3539. https://doi.org/10.1103/PhysRevE.47.3532

DOI:

10.1103/PhysRevE.47.3532

Document status and date:

Published: 01/05/1993

\section{Document Version:}

Publisher's PDF, also known as Version of Record (includes final page, issue and volume numbers)

\section{Please check the document version of this publication:}

- A submitted manuscript is the version of the article upon submission and before peer-review. There can be important differences between the submitted version and the official published version of record. People interested in the research are advised to contact the author for the final version of the publication, or visit the $\mathrm{DOI}$ to the publisher's website.

- The final author version and the galley proof are versions of the publication after peer review.

- The final published version features the final layout of the paper including the volume, issue and page numbers.

Link to publication

\section{General rights}

Copyright and moral rights for the publications made accessible in the public portal are retained by the authors and/or other copyright owners and it is a condition of accessing publications that users recognise and abide by the legal requirements associated with these rights.

- Users may download and print one copy of any publication from the public portal for the purpose of private study or research.

- You may not further distribute the material or use it for any profit-making activity or commercial gain

- You may freely distribute the URL identifying the publication in the public portal.

If the publication is distributed under the terms of Article 25fa of the Dutch Copyright Act, indicated by the "Taverne" license above, please follow below link for the End User Agreement:

www.tue.nl/taverne

Take down policy

If you believe that this document breaches copyright please contact us at:

openaccess@tue.nl

providing details and we will investigate your claim. 


\title{
Self-consistent cluster approach to the homogeneous kinetic nucleation theory
}

\author{
V. I. Kalikmanov* and M. E. H. van Dongen \\ Physics Department, Eindhoven University, Building W\&S, P.O. Box 513, 5600 MB, Eindhoven, The Netherlands
}

(Received 14 February 1992)

\begin{abstract}
An alternative, self-consistent formulation of the homogeneous nucleation theory has been proposed. This approach differs from the classical Becker-Döring-Zeldovich theory in two respects: (i) evaporation rates are evaluated by referring to the stable equilibrium of a saturated vapor rather than to the constrained metastable equilibrium of a supersaturated vapor; and (ii) for the reference stable equilibrium state the Fisher theory of condensation is used in order to obtain a self-consistent definition of the freeenergy barrier for $l$-cluster formation, where $l$ is the number of molecules in the cluster. A comparison of the expressions for the nucleation rate and critical cluster size with the corresponding classical expressions has been made for the different parts of the phase diagram (temperature-supersaturation) and the domain where both theories are close has been found. Predictions of the present theory have been compared with the experimental results on nucleation of $n$-nonane for the three sets of experiments (diffusion cloud chamber, fast-expansion cloud chamber, and two-piston cloud chamber). It has been shown that the present theory has a much better agreement with experimental results for $n$-nonane than the classical theory.
\end{abstract}

PACS number(s): 82.60.Nh, 64.60.Qb, 05.70.Fh

\section{INTRODUCTION}

The theory of homogeneous nucleation from a supersaturated vapor has been the subject of intense investigations since the 1930's. The approach developed in the early works by Becker and Döring [1] and Zeldovich [2] is now referred to as "classical nucleation theory." It is based on the assumption (known as "capillarity approximation") that the free energy of a cluster (droplet) is equal to the bulk free energy plus the free energy of the cluster surface. Classical theory results in a simple expression for the nucleation rate as a function of supersaturation and temperature. However, in a number of experiments it was found that predictions of classical theory differed from experimental values by several orders of magnitude [3-6].

Various modifications of the classical theory have been proposed. Lothe and Pound [7] suggested that a cluster should be treated as a large molecule and additional contributions to the free energy due to the translational and rotational degrees of freedom should be taken into account. Their calculations increased the nucleation rate by a factor of $10^{14}-10^{18}$. Reiss, Katz, and Cohen [8] criticized this idea (which, as they pointed out, led to the "translation-rotation paradox"), saying that it counted certain contributions to the free energy twice. Instead they proposed a model that assumed that the free energy of a cluster with fixed boundaries and a center of mass, which fluctuates over the entire cluster, was equal to the free energy of that same number of molecules in the bulk liquid plus a surface free energy without corrections due to translation and rotation. The result they obtained turned out to be close to the classical one. Several other attempts have been made (see, e.g., [9]) which were not fully satisfactory. So the classical theory still remains the most widely used method to predict nucleation rates.

The classical theory (as well as the above-mentioned models) represents a combination of kinetics of cluster growth and thermodynamics. In the kinetic part it assumes that cluster growth and decay are dominated by monomer addition (condensation) and monomer extraction (evaporation). For an ideal vapor the condensation rate is known from the gas kinetic theory. The evaporation rate is obtained by reference to the so-called "constrained steady state" (McDonald [10]), which would exist for a vapor at the same temperature and supersaturation $S_{\mu}>1$ as the vapor in question. Such a fictitious state was postulated in order to enable the integration of the flux equation determining the rate of nucleation. Constrained equilibrium is achieved by introducing Maxwell demons-monomers are continually replenished by the artificial dissociation of clusters which grow beyond a certain size. The necessity of such an artificial construction clearly follows the fact that a supersaturated vapor $\left(S_{\mu}>1\right)$ is not a true stable equilibrium but a nonequilibrium metastable state.

An alternative procedure was suggested by Katz and co-workers $[11,12]$ in which the evaporation rate was obtained by reference to the stable equilibrium of a saturated vapor $\left(S_{\mu}=1\right)$ at the same temperature as the vapor in question. Thus in this procedure, which we shall refer to as the "kinetic theory of nucleation" [11], no artificial construction is needed.

The crucial point of the theory is the choice of the model for a Gibbs free energy of cluster formation $\Delta G_{l}$. This choice constitutes the thermodynamic part of nucleation theory. Katz and co-workers used the classical, purely phenomenological definition of $\Delta G_{l}$, containing the surface term and the bulk term. Assuming clusters to be spherical, it reads

$$
\Delta G_{l} / k T=\theta l^{2 / 3}-l \ln S_{\mu},
$$

where $T$ is the temperature, $k$ is the Boltzmann constant, 
$\theta$ is a dimensionless surface tension, and $l$ is the number of molecules in the cluster. In case of the saturated vapor (which is the reference state in the kinetic approach of $[11,12])$ the bulk term vanishes and Eq. (1) results in

$$
\Delta G_{l} / k T=\theta l^{2 / 3} \text {. }
$$

It must be noted that although both approaches ([10] and [11]) use different reference states they yield the same expression for the nucleation rate and critical cluster size if for the Gibbs formation energy $\Delta G_{l}$ the phenomenological expression (1) is used. Predicted nucleation rates are typically too low at low temperatures and too high at high temperatures with errors on the order of 4-10 orders of magnitude in both directions.

As was pointed out by Blander and Katz in 1972 [13], the classical expression (1) is not self-consistent-a selfconsistent definition of $\Delta G_{l}$ should satisfy the condition $\Delta G_{1}=0$. In case of the kinetic approach this demand becomes particularly clear: $\Delta G_{1}$ represents the difference in chemical potential between a molecule in the vapor and a molecule in the condensed phase for a system at an equilibrium saturated state; then the condition of phase equilibrium implies that these chemical potentials are equal, thus $\Delta G_{1}=0$. This idea was used by Girshick and Chiu [14] who suggested writing $\Delta G_{l}$ in the kinetic approach in the form

$$
\Delta G_{l} / k T=\theta\left(l^{2 / 3}-1\right) .
$$

Such choice introduces an extra factor $e^{\theta}$ into the nucleation rate.

Another extension of classical nucleation theory has been suggested recently by Dillmann and Meier [15], who used the classical McDonald approach [10] and the semiphenomenological Fisher cluster theory of condensation [16] as an ansatz for the energy barrier $\Delta G_{l}$. Dillmann and Meier introduced a curvature correction factor to the surface tension

$$
\kappa(l)=1+\alpha_{1} l^{-1 / 3}+\alpha_{2} l^{-2 / 3},
$$

where $\alpha_{1}$ and $\alpha_{2}$ were temperature-dependent quantities selected to fit the saturated vapor pressure and the second virial coefficient. The parameter $\alpha_{1}$ defines the so-called Tolman length [17]. A lot of effort has been made to calculate $\alpha_{1}$ using molecular-dynamics simulations [18,19] of Lennard-Jones fluids but until now consensus has not been reached whether $\alpha_{1}$ has a finite or zero value. The most recent and extensive molecular-dynamics calculations of Nijmeijer et al. [19] indicate that within the error $\alpha_{1}$ cannot be distinguished from zero. This result has been confirmed by the recent analytical calculations of Blokhuis and Bedeaux [20]. They studied surface tension on the basis of the Irving-Kirkwood theory of a microscopic pressure tensor and came to the conclusion that for a symmetric density profile at the liquid-vapor interface the Tolman length is equal to zero.

Therefore instead of introducing curvature correction (3) it is reasonable to use the Fisher theory for deriving a self-consistent expression for the energy barrier (in the sense of Blander and Katz [13]) and combine it with the kinetic approach. This is the aim of the present paper.

\section{MODEL}

Our theory is based on the following assumptions. (i) Cluster growth and decay are dominated by monomer addition (condensation) and monomer extraction (evaporation); (ii) mass accommodation coefficients are equal to unity; (iii) there is no intercluster interaction (i.e., the excluded volume effects and long-range interaction terms are not taken into account); and (iv) the surface area of a $l$-cluster $s_{l}$ can be written in the form

$$
s_{l}=s_{1} l^{\sigma}, \quad 0<\sigma<1 .
$$

The parameter $\sigma$ characterizes the geometrical shape of the cluster. For spherical clusters $\sigma=\frac{2}{3}$.

Let $J_{l}$ be the net rate of $l$-cluster formation $\left(\mathrm{cm}^{-3} \mathrm{~s}^{-1}\right)$. Using assumptions (i) and (ii) it can be written in the form

$$
J_{l}=v s_{l-1} \rho_{l-1}-R_{l} \rho_{l},
$$

where $\rho_{l}$ is number density of $l$ clusters $\left(\mathrm{cm}^{-3}\right), v$ is the rate of impingement $\left(\mathrm{cm}^{-2} \mathrm{~s}^{-1}\right)$ representing monomer flux to the unit surface, $R_{l}$ is the evaporation rate of the $l$ cluster $\left(\mathrm{s}^{-1}\right)$. The rate of impingement is determined only by monomers in view of assumption (i). From the ideal gas kinetics [21],

$$
v=\rho_{1} \sqrt{k T / 2 \pi m_{1}}
$$

where $m_{1}$ is the mass of a molecule. In equilibrium $J_{l}=0$ for each $l$, so from (5),

$$
R_{l}=v^{0} s_{l-1} \frac{\rho_{l-1}^{0}}{\rho_{l}^{0}},
$$

where the superscript 0 denotes the true equilibrium state ("reference state") at the same temperature as the supersaturated state of interest. Following [12] we rewrite (4) in the form

$$
\frac{J_{l}}{v s_{l-1} \rho_{l-1}^{0}}=\frac{\rho_{l-1}}{\rho_{l-1}^{0}}-\frac{v^{0}}{v} \frac{\rho_{l}}{\rho_{l}^{0}},
$$

and introduce a supersaturation coefficient

$$
S_{\mu}=v / \nu^{0}=\rho_{1} / \rho_{1}^{0},
$$

characterizing the nonequilibrium state. Dividing both sides of Eq. (7) by $S_{\mu}^{l-1}$, we get

$$
\frac{J_{l}}{v s_{l-1} \rho_{l-1}^{0} S_{\mu}^{l-1}}=\frac{1}{S_{\mu}^{l-1}} \frac{\rho_{l-1}}{\rho_{l-1}^{0}}-\frac{1}{S_{\mu}^{l}} \frac{\rho_{l}}{\rho_{l}^{0}} .
$$

If we sum this equation from $l=2$ to an arbitrary large $L$ then successive terms in the right-hand side cancel, resulting in

$$
\sum_{l=2}^{L} \frac{J_{l}}{v S_{l-1} \rho_{l-1}^{0} S_{\mu}^{l-1}}=1-\frac{\rho_{L}}{S_{\mu}^{L} \rho_{L}^{0}} .
$$

The dynamics of the nucleation process is described by the kinetic equation

$$
\frac{\partial \rho_{l}}{\partial t}=J_{l-1}-J_{l}
$$

Since a steady state is rapidly achieved we are interested 
in the stationary solution when $J_{l}$ is constant, irrespective of cluster size $J_{l}=I$. The quantity $I$ is called a nucleation rate. As shown by $\mathrm{Katz}$ and Donohue [12] $\rho_{L}$ in Eq. (9) is a slowly decreasing function of $L$, whereas $S_{\mu}^{L} \rho_{L}^{0}$ is a rapidly increasing function of $L$. Therefore, for sufficiently large $L$ the second term on the right-hand side of Eq. (9) becomes negligible. Replacing summation by integration we obtain

$$
I=\left[\int_{0}^{\infty}\left(v s_{l} \rho_{l}^{0} S_{\mu}^{l}\right)^{-1} d l\right]^{-1} .
$$

By introducing an auxiliary function

$$
H(l)=\ln \left(v s_{l} \rho_{l}^{0} S_{\mu}^{l}\right),
$$

the integrand can be written in an exponential form:

$$
I=\left[\int_{0}^{\infty} e^{-H(l)} d l\right]^{-1} .
$$

Expanding $H(l)$ in a Taylor series around $l=l_{c}$, corresponding to a minimum of $H(l)$, and truncating after the quadratic term

$$
H(l) \cong H\left(l_{c}\right)+\frac{1}{2}\left(\frac{d^{2} H}{d l^{2}}\right]_{l=l_{c}}\left(l-l_{c}\right)^{2},
$$

we obtain for the nucleation rate:

$$
\left.I=\left[H^{\prime \prime}\left(l_{c}\right) / 2 \pi\right]^{1 / 2} \exp \left[H\left(l_{c}\right)\right] \quad \quad \quad \prime^{\prime \prime}=\frac{d^{2}}{d l^{2}}\right] .
$$

The quantity $l_{c}$ represents the critical cluster size.

In order to proceed it is necessary to specify the equilibrium number-density distribution $\rho_{l}^{0}$. In the absence of intercluster interaction [assumption (iii)] it can be expressed in the following form [16]:

$$
\rho_{l}^{0}=\frac{q_{l}}{V} z^{l}
$$

where $q_{l}$ is the configuration integral of the $l$ cluster, $V$ is the volume of the system, $z$ is the fugacity

$$
z=\exp \left(\beta \mu^{0}\right) / \lambda^{3},
$$

$\beta=1 / k T, \mu^{0}$ is the chemical potential in the equilibrium reference state, $\lambda=\left(2 \pi \hbar^{2} / m_{1} k T\right)^{1 / 2}$ is the thermal de Broglie wavelength. The Helmholtz free energy of the $l$ cluster $F_{l}$ reads

$$
\beta F_{l}=-\ln \left(q_{l} / \lambda^{3 l}\right) .
$$

Combining Eqs. (12)-(14), we obtain

$$
\rho_{l}^{0}=\frac{1}{V} \exp \left(-\beta \Omega_{l}\right)
$$

where

$$
\Omega_{l}=F_{l}-\mu^{0} l
$$

is the grand potential of an $l$ cluster. We can rewrite this result introducing the equilibrium number density of monomers $\rho_{1}^{0}$ :

$$
\rho_{l}^{0}=\rho_{1}^{0} \exp \left(-\beta \Delta \Omega_{l}\right),
$$

where

$$
\Delta \Omega_{l}=\Omega_{l}-\Omega_{1}
$$

plays the role of an energy barrier for $l$-cluster formation (or activation energy). It can be shown on the basis of simple thermodynamic arguments [22] that $\Delta \Omega_{l}$ is equal to the Gibbs free formation energy $\Delta G_{l}$ used in classical theory. The configuration integral derived by Fisher [16] on the basis of assumptions (iii) and (iv) reads

$$
\begin{aligned}
q_{l}= & V\left\{\exp \left[-\beta \mu_{\sigma}+\ln \lambda^{3}\right]\right\}^{l} \\
& \times\left\{\exp \left[-\beta s_{1} \gamma_{0}\left(1-T / T_{c}\right)\right]\right\}^{l^{\sigma}} l^{-\tau} q_{0},
\end{aligned}
$$

where $\mu_{\sigma}(T)$ is the chemical potential at the saturation point (i.e., on the binodal),

$$
\gamma=\gamma_{0}\left(1-T / T_{c}\right)
$$

is the "microscopic" surface tension, $T_{c}$ is the critical temperature, and $q_{0}$ and $\tau$ are constants related to the parameters of the critical state. Parameter $\tau$ satisfies the equation [23]

$$
Z_{c} \equiv p_{c} / \rho_{c} k T_{c}=\zeta(\tau) / \zeta(\tau-1),
$$

where $p_{c}$ and $\rho_{c}$ are the critical pressure and critical number density, respectively, $\zeta(x)=\sum_{i=1}^{\infty} i^{-x}$ is the Riemann $\zeta$ function. $\tau$ can vary in the limits: $2<\tau<3$. Using thermodynamic relations (14) and (15) we find for the energy barrier

$$
\beta \Delta \Omega_{l}=\theta\left(l^{\sigma}-1\right)+\tau \ln l-\beta\left(\mu^{0}-\mu_{\sigma}\right)(l-1),
$$

where

$$
\theta(T)=\beta s_{1} \gamma
$$

is the dimensionless surface tension. Introducing a dimensionless temperature $t=T / T_{c}$, we can rewrite it as

where

$$
\theta=\theta_{c}(1 / t-1),
$$

$$
\theta_{c}=s_{1} \gamma_{0} / k T_{c}
$$

characterizes a particular substance. The quantity $s_{1}$ may be calculated, assuming sphericity of a monomer, given molecular weight $M(\mathrm{~g} / \mathrm{mol})$ and mass density in the liquid phase $\rho_{\text {liq }}\left(\mathrm{g} / \mathrm{cm}^{3}\right): s_{1}=\left(6 \sqrt{\pi} m_{1} / \rho_{\text {liq }}\right)^{2 / 3}$; $m_{1}=M / N_{A}\left(N_{A}\right.$ is the Avogadro constant).

Until now we have not yet specified the equilibrium reference state characterized by the chemical potential $\mu^{0}$ $\left[\mu^{0} \leq \mu_{\sigma}(T)\right]$. It is convenient to choose as this state the saturation point at given temperature $T$ (although any other point on the corresponding gaseous isotherm could be used). Then $\mu^{0}=\mu_{\sigma}(T)$ and

$$
\beta \Delta \Omega_{l}=\theta\left(l^{\sigma}-1\right)+\tau \ln l .
$$

One can see that the energy barrier defined by Eq. (22) vanishes at $l=1$, satisfying therefore the condition of self-consistency [13]. From (16) and (22) the equilibrium number-density distribution reads

$$
\rho_{l}^{0}=\rho_{1}^{0} \exp \left[-\theta\left(l^{\sigma}-1\right)-\tau \ln l\right] .
$$

Substituting this result into the function $H(l)$ defined in Eq. (10), we obtain 
$H(l)=\ln \left(v \rho_{1}^{0} s_{1}\right)+\ln l^{\sigma}-\theta\left(l^{\sigma}-1\right)-\tau \ln l+l \ln S_{\mu}$.

Differentiation of $H(l)$ with respect to $l$ gives the equation for the critical cluster size $l_{c}$ :

$$
\sigma \theta l_{c}^{\sigma}-l_{c} \ln S_{\mu}=-(\tau-\sigma) .
$$

It has a unique solution for each value of the supersaturation $S_{\mu}>1$. Near the binodal (where $S_{\mu} \rightarrow 1$ ) $l_{c}$ tends to infinity. On the other hand the restriction $l_{c} \geq 1$ gives, somewhat arbitrarily, the extreme "limit of metastability" $S_{\mu}^{\max }$ :

$$
S_{\mu}^{\max }(T)=\exp [\sigma \theta(T)+\tau-\sigma] .
$$

Values of $\theta(T)$ for different substances range within wide limits: from $\sim 3$ for methanol at $350 \mathrm{~K}$ to $\sim 50$ for mercury at $290 \mathrm{~K}$ [14]. Thus $S_{\mu}^{\max }$ varies from $\sim e^{2}$ to $\sim e^{53}$.

The second derivative of $H$ at $l=l_{c}$,

$$
H^{\prime \prime}\left(l_{c}\right)=\frac{1}{l_{c}^{2}}\left[\sigma(1-\sigma) \theta l_{c}^{\sigma}+\tau-\sigma\right],
$$

is positive corresponding to a minimum of $H$ as expected. Combining Eqs. (10), (11), (23), and (26), we obtain for the nucleation rate

$$
\begin{aligned}
I= & \nu s_{1} \rho_{1}^{0} S_{\mu}^{l_{c}} l_{c}^{-(1+\tau-\sigma)}\left[\frac{\sigma(1-\sigma) \theta l_{c}^{\sigma}+\tau-\sigma}{2 \pi}\right]^{1 / 2} \\
& \times \exp \left[-\theta\left(l_{c}^{\sigma}-1\right)\right],
\end{aligned}
$$

with the critical cluster size $l_{c}$ given by Eq. (25).

\section{PHASE DIAGRAM}

In order to compare the results obtained with those of the classical theory we rewrite Eq. (25) in the form

$$
\sigma \theta l_{c}^{\sigma}\left[1+\frac{\tau-\sigma}{\sigma \theta l_{c}^{\sigma}}\right]=l_{c} \ln S_{\mu},
$$

where we set $\sigma=\frac{2}{3}$ (spherical droplets). For sufficiently large $l_{c}$ the second term in brackets becomes negligible and the solution reads

$$
l_{c}=\left[\frac{2}{3}\left(\theta / \ln S_{\mu}\right)\right]^{3},
$$

which is identical to the critical size in the classical theory. In the present theory this result is valid provided that $\left(\tau-\frac{2}{3}\right) /\left(\frac{2}{3} \theta l_{c}^{2 / 3}\right)<<1$. In order to use this solution in the calculation of the nucleation rate given by Eq. (27) we need a slightly stronger inequality, namely, $\left(\tau-\frac{2}{3}\right) /\left(\frac{2}{9} \theta l_{c}^{2 / 3}\right)<<1$ which after substitution of Eq. (28) gives

$$
\frac{81}{8} \frac{(\tau-2 / 3) \ln ^{2} S_{\mu}}{\theta^{3}} \ll 1 .
$$

Provided this inequality is valid we obtain the following result:

$$
I=f_{\tau} I_{\mathrm{cl}},
$$

where

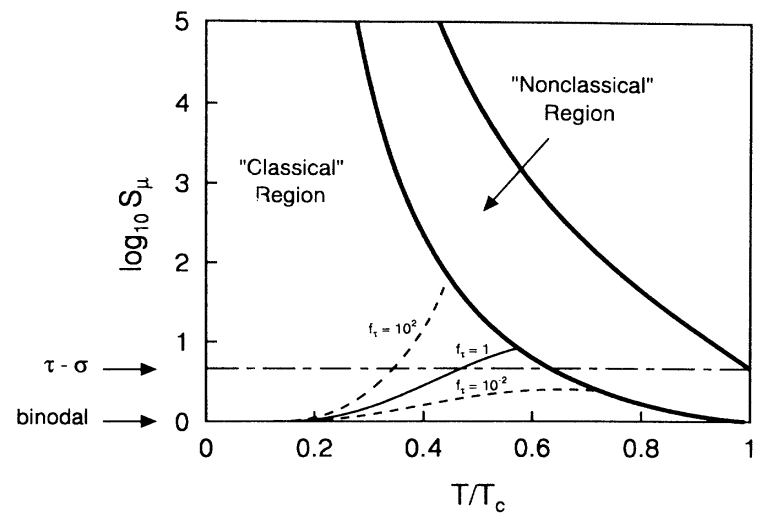

FIG. 1. Phase diagram. The data used are those of $n$-nonane (see the text).

$$
I_{\mathrm{cl}}=v s_{1} \rho_{1} \frac{1}{3} \sqrt{\theta / \pi} \exp \left[-\frac{4}{27} \frac{\theta^{3}}{\ln ^{2} S_{\mu}}\right]
$$

is the classical result for the nucleation rate and

$$
f_{\tau}\left(\theta(T), S_{\mu}\right)=\frac{e^{\theta}}{S_{\mu}}\left[\frac{3}{2} \frac{\ln S_{\mu}}{\theta}\right]^{3 \tau} .
$$

Thus in the "classical" region [corresponding to inequality (29)] of the phase diagram $\left(T-S_{\mu}\right)$ (Fig. 1) the present theory and the classical one predict the same result for the size of the critical nucleus, whereas the nucleation rates differ by a factor $f_{\tau}$. [Note that by setting formally $\tau=0$ one comes to the result $I=\left(e^{\theta} / S_{\mu}\right) I_{\mathrm{cl}}$, which Girshick and Chiu [14] have obtained recently on the basis of phenomenological considerations. However the value $\tau=0$ is beyond the range of validity of the present theory, which is $2<\tau<3$.] Within the "classical" region one can find an area where the difference between $I$ and $I_{\mathrm{cl}}$ is not large. It depends on the value of $f_{\tau}$; e.g., within the area bounded by the lines $f_{\tau}=10^{2}$ and $f_{\tau}=10^{-2}$ this difference is not more than 2 orders of magnitude.

In the "nonclassical" region of the phase diagram, limited from above by the maximal value of supersaturation at given temperature $S_{\mu}^{\max }(T)$, relation (30) does not hold.

\section{RESULTS AND DISCUSSION}

In order to calculate the nucleation rate from Eq. (27) and compare it with available experimental data one must define the nonequilibrium number density of monomers $\rho_{1}$. In case of nucleation from the vapor $\rho_{1}$ is much greater than the sum of number densities of all other $l$ clusters, $l=2,3, \ldots$ [24]. Thus one can derive $\rho_{1}$ from the vapor pressure via the relation $p \cong \rho_{1} k T$. Combining it with Eqs. (6), (8), and (27) we obtain

$$
\begin{aligned}
I= & \frac{s_{1} p_{\text {sat }}^{2}}{k T \sqrt{2 \pi m_{1} k T}} S_{\mu}^{l_{c}+1} l_{c}^{-(1+\tau-\sigma)} \\
& \times\left[\frac{\sigma(1-\sigma) \theta l_{c}^{\sigma}+\tau-\sigma}{2 \pi}\right]^{1 / 2} \exp \left[-\theta\left(l_{c}^{\sigma}-1\right)\right],
\end{aligned}
$$


where $p_{\text {sat }}(T)$ is a saturated vapor pressure at temperature $T$.

We compare predictions of the present theory with the experimental results for $n$-nonane $\left(\mathrm{C}_{9} \mathrm{H}_{20}\right)$ for which reliable experimental data exists and with predictions of the classical theory. During the last decade new experimental techniques have been developed which make it possible to measure directly the nucleation rate as a function of supersaturation and temperature in the different domains of the phase diagram. $n$-nonane has been studied by three research groups-Hung, Krasnopoler, and Katz [6], Adams, Schmitt, and Zalabsky [3], and Wagner and Strey [27]-and consistent results have been observed. Hung, Krasnopoler, and Katz [6] used the thermal-diffusion cloud chamber and made measurements in the range of low to intermediate rates $\left(10^{-5} \mathrm{~cm}^{-3} \mathrm{~s}^{-1} I<10^{2} \mathrm{~cm}^{-3} \mathrm{~s}^{-1}\right)$. A second technique, which uses a fast-expansion piston cloud chamber, was applied by Adams, Schmitt, and Zalabsky [3] in the range of intermediate to high rates $\left(10^{2} \mathrm{~cm}^{-3} \mathrm{~s}^{-1}<I<10^{5}\right.$ $\left.\mathrm{cm}^{-3} \mathrm{~s}^{-1}\right)$ and by Wagner and Strey [27] in the range of high rates $\left(10^{6} \mathrm{~cm}^{-3} \mathrm{~s}^{-1}<I<10^{10} \mathrm{~cm}^{-3} \mathrm{~s}^{-1}\right.$ ) (in [27] the two-piston expansion cloud chamber was used). We compare our theoretical results with these three sets of experiments.

The following values of the physical properties are used in our calculations [25]:

$$
\begin{aligned}
M= & 128.259 \mathrm{~g} / \mathrm{mol}, \\
T_{c}= & 594.6 \mathrm{~K}, \quad Z_{c} \equiv p_{c} / \rho_{c} k T_{c}=0.26, \\
\rho_{\text {liq }}= & 0.733503-7.87562 \times 10^{-4} T_{\text {Cels }} \\
& -9.68937 \times 10^{-8} T_{\text {Cels }}^{2} \\
& -1.29616 \times 10^{-9} T_{\text {Cels }}^{3} \mathrm{~g} / \mathrm{cm}^{3},
\end{aligned}
$$

where $T_{\text {Cels }}=T-273.15$.

For $p_{\text {sat }}(T)$ we use the King-Najjar equation [26]

$$
\begin{aligned}
p_{\text {sat }}(T)=\exp [ & -17.56832 \ln T+1.52556 \times 10^{-2} T \\
& -9467.4 / T+135.974] \mathrm{dyn} / \mathrm{cm}^{2},
\end{aligned}
$$

which most accurately fits experimental data on equilibrium properties of $n$-nonane [6]. The solution of Eq. (20) reads $\tau=2.190$; for $\sigma$ the classical value $\sigma=\frac{2}{3}$ is used.

The best available surface tension is well described by the linear relation (Jasper isobaric equation) [28,29]

$$
\gamma_{\mathrm{C}_{9} \mathrm{H}_{20}}=a-b T
$$

where $a=50.2513 \mathrm{dyn} / \mathrm{cm}$ and $b=0.09347 \mathrm{dyn} / \mathrm{cm} \mathrm{K}$. We must note that all the measurements of surface tension were made at $-20^{\circ} \mathrm{C}$ and higher [28]. That is why in order to cover the whole range of nucleation data from $T_{0}=203 \mathrm{~K}$ in experiments of Wagner and Strey [27] up to $T_{f}=315 \mathrm{~K}$ in experiments of Hung, Krasnopoler, and Katz [6], we have to extrapolate the linear law (33) to the lower range of temperatures. As was pointed out in [6] the total uncertainty in surface tension is no more than $0.5-1 \%$ over the temperature range $\left[T_{0}, T_{f}\right]$. It is this relation (33) and its extrapolation to lower temperatures

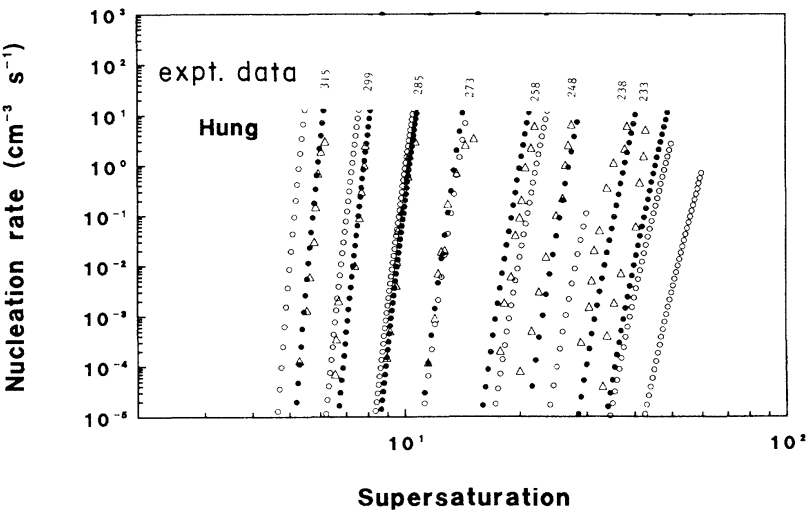

FIG. 2. Comparison of experimental nucleation rates for $n$ nonane with the predictions of the classical theory (open circles) and the new theory (closed circles). Triangles, experimental data of Hung, Krasnopoler, and Katz [6]. The labels are temperatures in $\mathrm{K}$.

which is used in the calculation of the nucleation rate in the classical theory [Eq. (31)].

The present theory in its equilibrium thermodynamic part is based on the Fisher cluster model in which the surface tension is given by Eq. (19). This form of temperature dependence is essential for the relations between critical indexes. Therefore in order for our theory to be consistent we have to use Eq. (19) for $\gamma$. The unknown free parameter $\gamma_{0}$ can be then found with the help of the best-fit procedure (linear regression) given the experimental data on surface tension [Eq. (33)] over the temperature range $\left[T_{0}, T_{f}\right]$. It results in

$\gamma_{0}=\int_{T_{0}}^{T_{f}}(a-b T)\left(1-T / T_{c}\right) d T / \int_{T_{0}}^{T_{f}}\left(1-T / T_{c}\right)^{2} d T$.

Integration gives $\gamma_{0}=46.224 \mathrm{dyn} / \mathrm{cm}$.

In Fig. 2 experimental data of Hung, Krasnopoler, and

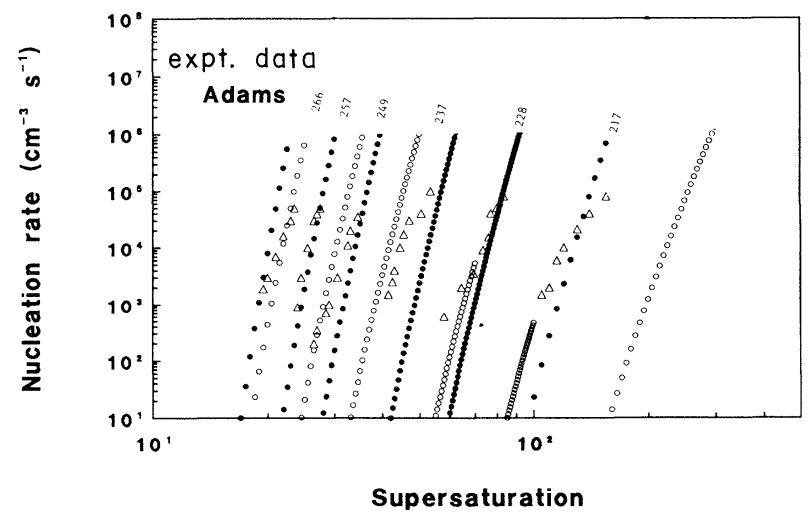

FIG. 3. Comparison of experimental nucleation rates for $n$ nonane with the predictions of the classical theory (open circles) and the new theory (closed circles). Triangles, experimental data of Adams, Schmitt, and Zalabsky [3]. The labels are temperatures in $\mathbf{K}$. 


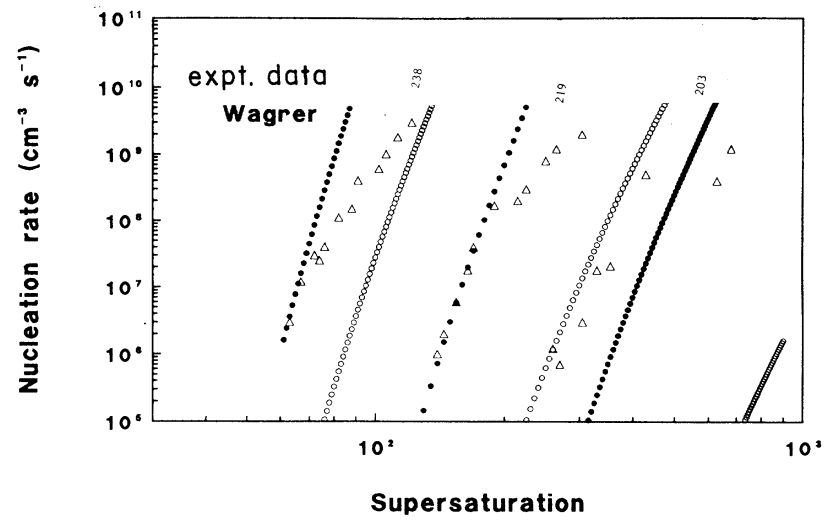

FIG. 4. Comparison of experimental nucleation rates for $n$ nonane with the predictions of the classical theory (open circles) and the new theory (closed circles). Triangles, experimental data of Wagner and Strey [27]. The labels are temperatures in K.
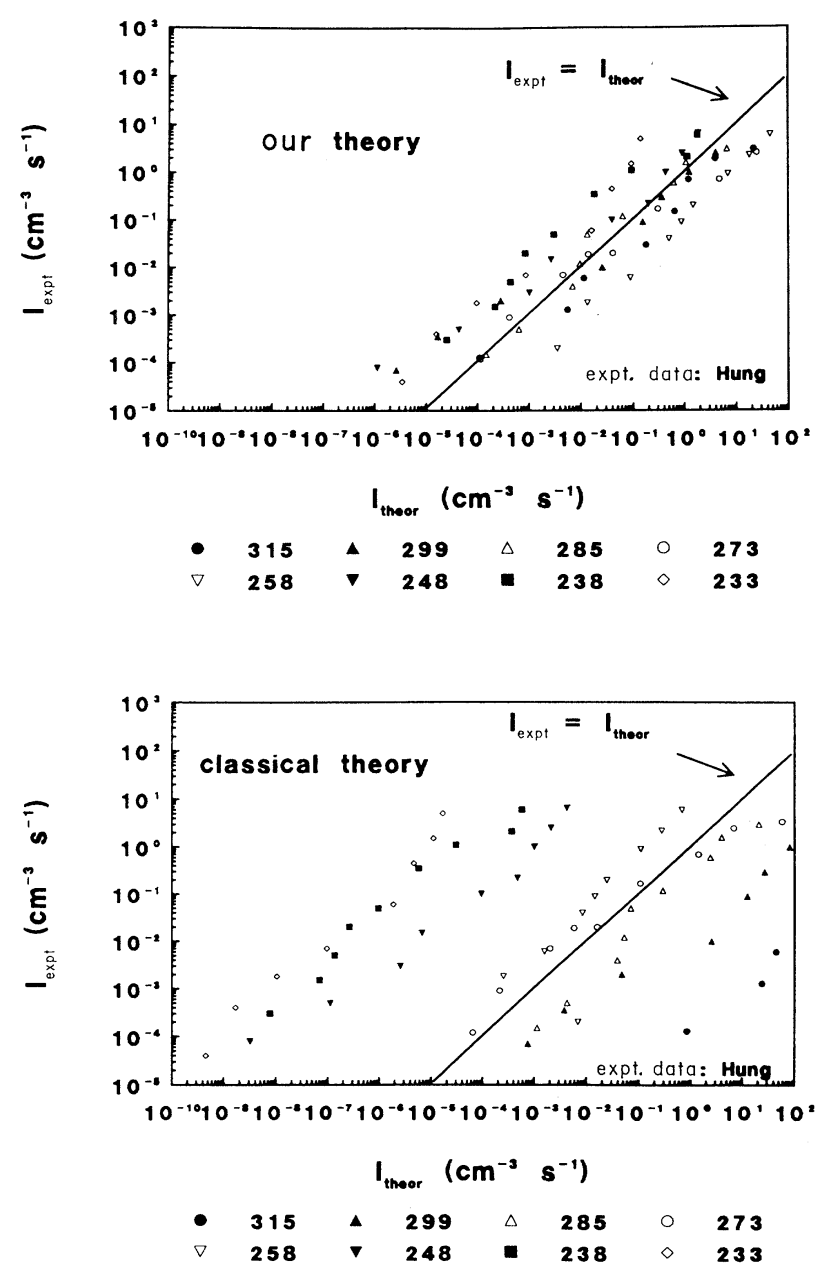

FIG. 5. Nucleation rate: experiment of Hung, Krasopoler, and Katz [6] vs theory (classical theory, lower part; new theory, upper part). The labels are temperatures in $\mathrm{K}$.
Katz [6] are compared to the predictions of classical theory and of our alternative theory in an $\left(I-S_{\mu}\right)$ plot for different temperatures; labels correspond to temperatures in $\mathrm{K}$. Figures 3 and 4 do the same with the experimental data of Adams, Schmitt, and Zalabsky [3] and Wagner and Strey [27], respectively. It can be seen that the classical theory gives satisfactory predictions in a rather narrow temperature range, approximately between 258 and $285 \mathrm{~K}$. For $T>285 \mathrm{~K}$ classical results are 3-4 orders of magnitude higher than experimental nucleation rates. For $T<258 \mathrm{~K}$ the tendency is the reverse: classical results are 3-8 orders of magnitude lower than experimental data. The present theory gives much better predictions in the whole temperature range studied except for the measurements with high nucleation rates $\left(T \geq 10^{8}\right.$ $\mathrm{cm}^{3} \mathrm{~s}^{-1}$ ) in the experiments of Wagner and Strey; for $I \geq 10^{8} \mathrm{~cm}^{-3} \mathrm{~s}^{-1}$ our theoretical results are $2-4$ orders of magnitude higher than the experimental data.

In order to make a comparison between different theories and experiment more vivid we show in Figs. 5-7 a plot of experimental data versus predictions of theory for different temperatures. Perfect agreement between theory and experiment would be demonstrated if all the data fell on the same ("ideal") line $I_{\text {expt }}=I_{\text {theor }}$. The best agreement between the alternative theory and observations is obtained for the experiment of Hung, Krasnopoler, and Katz (Fig. 5).

In Fig. 8 the ratio of critical cluster sizes $\left(l_{c, \mathrm{cl}} / l_{c}\right)$
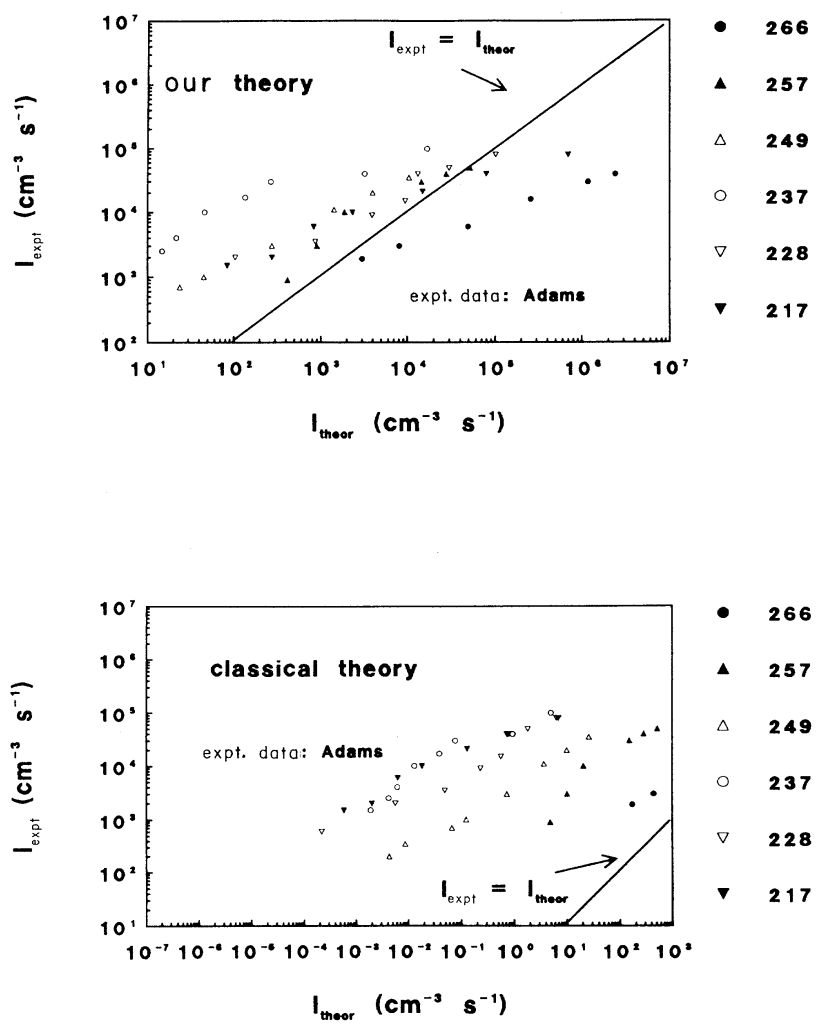

FIG. 6. Nucleation rate: experiment of Adams, Schmitt, and Zalabsky [3] vs theory (classical theory, lower part; new theory, upper part). The labels are temperatures in $\mathbf{K}$. 

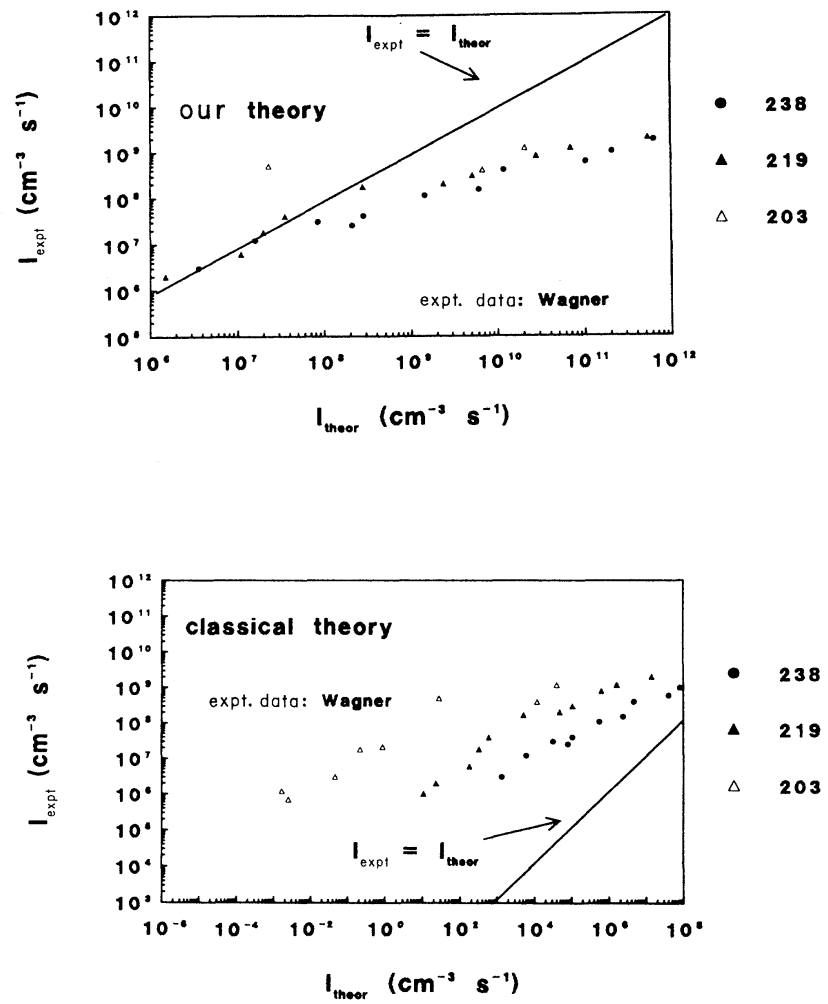

FIG. 7. Nucleation rate: experiment of Wagner and Strey [27] vs theory (classical theory, lower part; alternative theory, upper part). The labels are temperatures in $\mathrm{K}$.

versus temperature is shown for different values of supersaturation. For low temperatures this ratio weakly depends on supersaturation and is close to unity. For high temperatures $l_{c, \mathrm{cl}} / l_{c}<1$ and strongly depends on supersaturation.

In conclusion, we have presented an alternative formulation of the homogeneous nucleation theory which

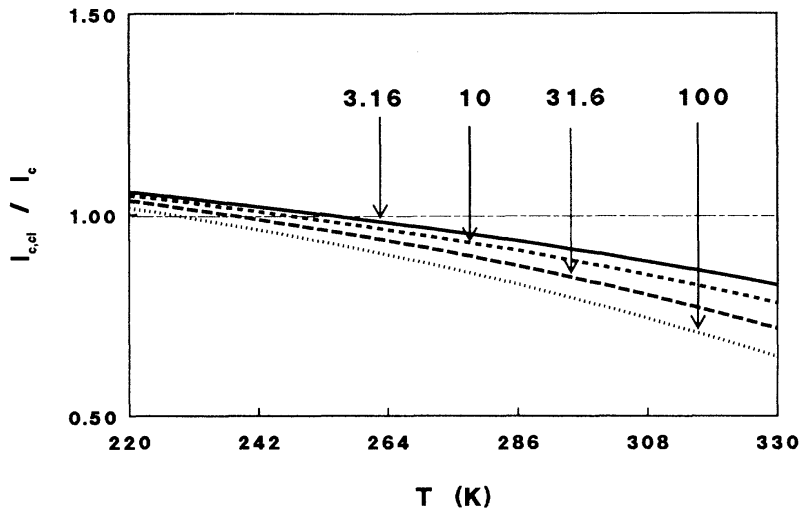

FIG. 8. Ratio of the critical cluster sizes vs temperature. $l_{c, \mathrm{cl}}$ classical value; $l_{c}$, alternative theory. The labels correspond to the values of supersaturation.

differs from the classical Becker-Döring-Zeldovich theory in two respects: (i) evaporation rates are evaluated by referring to the stable equilibrium of a saturated vapor rather than to the constrained metastable equilibrium of a supersaturated vapor; and (ii) for the reference stable equilibrium state the Fisher theory of condensation is used in order to obtain a self-consistent definition (in the sense of Blander and Katz [13]) of the energy barrier for $l$-cluster formation. We compared it with the three sets of experiments on $n$-nonane and showed that it agrees much better with experimental results than the classical nucleation theory.

\section{ACKNOWLEDGMENTS}

The authors thank G. Hofmans and K. Looijmans for critical remarks and assistance in preparing the manuscript. This work was financially supported by the Dutch Foundation for Fundamental Research on Matter (FOM).
*On leave from Institute For High Temperatures, Izhorskaya street 13/19, Moscow, 127412, Russia.

[1] R. Becker and W. Döring, Ann. Phys. (Leipzig) 24, 719 (1935).

[2] Ya. B. Zeldovich, Acta Physicochim. URSS 18, 1 (1943).

[3] G. W. Adams, J. L. Schmitt, and R. A. Zalabsky, J. Chem. Phys. 81, 5074 (1984).

[4] R. Strey, P. E. Wagner, and T. Schmeling, J. Chem. Phys. 84, 2325 (1986)

[5] A. Kacker and R. H. Heist, J. Chem. Phys. 82, 2734 (1985).

[6] C. Hung, M. Krasnopoler, and J. L. Katz, J. Chem. Phys. 90, 1856 (1989); 92, 7722 (1990).

[7] J. Lothe and G. M. Pound, J. Chem. Phys. 36, 2080 (1962).

[8] H. Reiss, J. L. Katz, and E. R. Cohen, J. Stat. Phys. 2, 83 (1968).

[9] Nucleation Phenomena, edited by A. C. Zettlemoyer (E1sevier, New York, 1977).

[10] J. E. McDonald, Am. J. Phys. 30, 870 (1962); 31, 31 (1963).

[11] J. L. Katz and H. Wiedersich, J. Colloid Interface Sci. 61,
351 (1977).

[12] J. L. Katz and M. D. Donohue, Adv. Chem. Phys. 40, 137 (1979).

[13] M. Blander and J. L. Katz, J. Stat. Phys. 4, 55 (1972).

[14] S. L. Girshick and C.-P. Chiu, J. Chem. Phys. 93, 1273 (1990).

[15] A. Dillmann and G. E. A. Meier, Chem. Phys. Lett. 160, 71 (1989); J. Chem. Phys. 94, 3872 (1991).

[16] M. E. Fisher, Physics 3, 255 (1967).

[17] R. C. Tolman, J. Chem. Phys. 17, 333 (1949).

[18] S. M. Thompson et al., J. Chem. Phys. 81, 530 (1984).

[19] M. Nijmeijer et al., J. Chem. Phys. 96, 565 (1992).

[20] E. M. Blokhuis and D. Bedeaux (unpublished).

[21] L. D. Landau and E. M. Lifshitz, Statistical Physics (Pergamon, Oxford, 1968).

[22] D. W. Oxtoby and R. Evans, J. Chem. Phys. 89, 7521 (1988).

[23] C. S. Kiang, Phys. Rev. Lett. 24, 47 (1970).

[24] J. P. Hirth and G. M. Pound, Prog. Mater. Sci. 11, 1 (1963). 
[25] R. Reid, J. Prausnitz, and B. Polling, The Properties of Gases and Liquids, 4th ed. (McGraw-Hill, New York, 1987).

[26] M. B. King and H. A. Najjar, Chem. Eng. Sci. 29, 1003 (1974).

[27] P. E. Wagner and R. Strey, J. Chem. Phys. 80, 5266 (1984).
[28] Selected Values of Properties of Hydrocarbons and Related Compounds, Thermodynamics Research Center (Texas A\&M University, College Station, TX, 1968).

[29] J. J. Jasper and E. V. Kring, J. Phys. Chem. 59, 1019 (1955). 